\title{
Performance of Composite Membrane for Palm Oil Wastewater Treatment
}

\author{
E. Yuliwati ${ }^{\mathrm{a}^{*}}$, H. Porawati ${ }^{\mathrm{b}}$, Elfidiah $^{\mathrm{a}}$ \& A. Melani ${ }^{\mathrm{a}}$

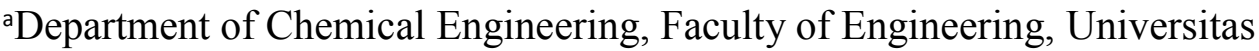 \\ Muhammadiyah Palembang, Indonesia, Jalan Ahmad Yani 13 Ulu Palembang 30263 \\ ${ }^{b}$ Department of Mechanical Engineering, Politeknik Jambi, Indonesia \\ Jalan Lingkar Barat 2, Lrg Veteran Bagan Pete, Kota Baru Jambi 36129
}

Submitted: 22/12/2018. Revised edition: 17/2/2019. Accepted: 19/2/2019. Available online: 15/7/2019

\begin{abstract}
Polyvinylidene fluoride (PVDF) composite membranes were prepared via phase inversion method by dispersing titanium dioxide $\left(\mathrm{TiO}_{2}\right)$ in the dope solution. It is aimed to study the effects of $\mathrm{TiO}_{2}$ on the membrane surface properties and thus its separation performance. The PVDF membranes modified by various $\mathrm{TiO}_{2}$ contents were characterized with respect to cross-sectional structure, average pore size, effective porosity, hydrophilicity, permeability and rejection. Maximum values of membrane hydrophilicity, membrane porosity and average pore size were achieved upon addition of $1.95 \% \mathrm{TiO}_{2}$. It is found that changes in the membrane surface properties are closely corresponded with the membrane fouling. The maximum flux and rejection of organic pollutants from palm oil wastewater were recorded at $82.5 \mathrm{~L} / \mathrm{m}^{2} \mathrm{~h}$ and $98.8 \%$, respectively using PVDF composite membrane incorporated with $1.95 \% \mathrm{TiO}_{2}$.
\end{abstract}

Keywords: Composite membrane, nanoparticle, palm oil wastewater, surface hydrophilicity

\subsection{INTRODUCTION}

Membrane technologies have extensively used in separation facilities to separate liquid/liquid or liquid/solid mixtures [1]. Polyvinylidene fluoride (PVDF) regarded as one of the most attractive polymer materials in microporous membrane industry. The molecular structure of PVDF homopolymer with alternating $\mathrm{CH}_{2}$ and $\mathrm{CF}_{2}$ groups along the polymer chain forms a unique polymer. It provides extraordinary mechanical properties, high chemical resistance, good thermal stability and excellent membrane forming abilities [2, 3]. Therefore, PVDF is a suitable material to make membranes, which applied in lithium ion batteries, pervaporation, wastewater treatment, etc. They can also be used ascomposites with inorganic nanoparticles such as $\mathrm{ZrO}_{2}$ [3], $\mathrm{SiO}_{2}[4,5]$, and $\mathrm{TiO}_{2}[6-8]$, to improve the membrane separation performance, thermal ability, chemical stability, and membrane forming ability by the combination of the basic properties of organic and inorganic materials. Among different inorganic nanoparticles, $\mathrm{TiO}_{2}$ has received the most attention because of its stability under harsh conditions, commercial availability, and easiness of preparation. When dispersed to PVDF membrane, $\mathrm{TiO}_{2}$ nanoparticles cannot only increase the hydrophilicity of membrane to enhance the flux and mitigate the fouling problem of PVDF membrane in MBR system $[9,10]$. The 
improvement of PVDF composite membrane properties can be achieved via sol-gel method, grafting, and physical blending of inorganic materials for its convenient operation [11].

Among these methods, physical blending is most interesting, owing to its convenient operation under mild conditions, and good performances of the resulting membranes [12, 13]. Yeow et al. [14] reported that $\mathrm{PVDF} / \mathrm{SiO}_{2}$ and $\mathrm{PVDF} / \mathrm{TiO}_{2}$ composite hollow fiber membranes could result in better performance, especially in terms of membrane structure, mechanical strength, and permeation properties. Cao et al. [15] and Wang and Kim [16] have studied the preparation of $\mathrm{TiO}_{2} / \mathrm{Al}_{2} \mathrm{O}_{3}$ composite hollow fiber membranes that exhibited better performances than the membranes with individually added $\mathrm{TiO}_{2}$ or $\mathrm{Al}_{2} \mathrm{O}_{3}$ nanoparticles. At present time, there are still few papers reporting the preparation and characterization of PVDF/multinanoparticles composite membranes, which might combine the advantages of polymer and various nanoparticles. Fontananova et al. [17] investigated the effect of nanoparticle additives, i.e., $\mathrm{TiO}_{2}, \quad$ on the thermodynamic/kinetic relations during the phase inversion process in the preparation of PVDF-based membranes. These additives were soluble in both DMAc and $\mathrm{H}_{2} \mathrm{O}$, and leached out of the solution during phase inversion process. The macrovoids became more accentuate and extend over the whole crosssection when $\mathrm{TiO}_{2}$ was present in the casting solution. The unique electronic, magnetic, and optical properties of nanoparticles improved the properties of polymer to a certain extent because of their small sizes, large surface areas, and strong activities [18]. The presence of finely dispersed inorganic particles in the polymer matrix has proven very useful in the improvement of membrane performance [19].

In this study, the fabrication of PVDF hollow fiber composite membranes by adding $\mathrm{LiCl} . \mathrm{H}_{2} \mathrm{O}$ and $\mathrm{TiO}_{2}$ in various concentrations was investigated, aiming to modify membrane surface properties and improve the filtration performance. Hydrophilicity of membranes usually expresses in terms of contact angle for a liquid drop on the membrane surface to measure the tendency for liquid to wet of the membrane surface. Pore size and porosity analysis of the PVDF hollow fiber composite membranes were investigated.

The surface and inner structures of the sample membranes were studied by FESEM. The performance for palm oil wastewater treatment was characterized by pure water flux and rejection efficiency of palm oil wastewater at various $\mathrm{pH}$ values of feed solution.

\subsection{METHODS}

\subsection{Materials}

Hollow fiber composite membranes were prepared using $\mathrm{Kynar}^{\circledR} 740$ PVDF polymer pellets purchased from Arkema Inc. Philadelphia, USA. DMAc (Synthesis Grade, Merck, $>99 \%$ ) was used as solvent without further purification. Lithium chloride monohydrate and titanium dioxide nanoparticles were used as inorganic additives (Sigma Aldrich). Glycerol (Merck Germany) was used as nonsolvent or the post treatment of membrane. In all cases, tap water was used as the external coagulation bath medium in the spinning process. 


\subsection{Preparation of Composite PVDF Spinning Dopes}

Pre-dried $\left(24 \mathrm{~h}\right.$ oven dried at $\left.50{ }^{\circ} \mathrm{C}\right)$ PVDF pellets was weighed and poured into pre-weighed DMAc solvent. The mixture was stirred to ensure thorough wetting of polymer pellets, prior to the addition of appropriate amounts of $\mathrm{TiO}_{2}$ at $50{ }^{\circ} \mathrm{C}$ to the mixture, which was continuously stirred for $48 \mathrm{~h}$ (IKA-20-W) at $500 \mathrm{rpm}$ until a homogenous solution, was formed. The polymer solution as kept by a glass bottle and air bubbles formed in the dope removed using water aspirator for several hours. The fully dissolved polymer solution transferred by a stainless-steel reservoir and left in the reservoir for $24 \mathrm{~h}$ at room temperature for degassing prior to spinning process. Solution viscosity measured using rheometer (Bohlin Instrument Ltd.) at various temperatures between 25 and $50{ }^{\circ} \mathrm{C}$.

\subsection{Fabrication of Composite Membrane}

The hollow fiber spinning process by dry-jet wet phase inversion was described elsewhere [20]. The details of the spinning dope compositions and spinning parameters are listed in Table 1 and 2 , respectively.

Table 1 Spinning dope compositions

\begin{tabular}{lcc}
\hline Sample & PVDF wt. $\%$ & TiO $_{2}$ wt. $\%$ \\
\hline PTL-0 & 19 & 0 \\
PTL-10 & 19 & 1.95 \\
PTL-20 & 19 & 3.8 \\
\hline
\end{tabular}

Briefly, the polymer solution was pressurized through the spinneret at a controlled extrusion rate, while internal coagulant was adjusted at $1.4 \mathrm{~mL} / \mathrm{min}$. The hollow fiber that had emerged from the tip of the spinneret was guided through the two water baths at a take up velocity $13.7 \mathrm{~cm} / \mathrm{s}$, carefully adjusted to match free falling velocity, before landing in a final collection bath to complete the solidification process. The spun hollow fibers were immersed in the water bath for a period of 3 days, with daily change of the water, to remove the residual DMAc and the additives. After the fibers were dried for 3 days, they were ready for making hollow fiber test modules.

Table 2 Spinning condition of PVDF hollow fiber membranes

\begin{tabular}{lll}
\hline $\begin{array}{l}\text { Dope extrusion rate } \\
(\mathrm{mL} / \mathrm{min})\end{array}$ & 4.20 \\
$\begin{array}{l}\text { Bore fluid } \\
\text { Bore fluid flow rate } \\
(\mathrm{mL} / \mathrm{min})\end{array}$ & 1.40 \\
$\begin{array}{l}\text { External coagulant } \\
\mathrm{H}_{2} \mathrm{O}\end{array}$ & & Tap water \\
$\begin{array}{l}\text { Air gap distance }(\mathrm{cm}) \\
\text { Spinneret o.d./i.d. }(\mathrm{mm})\end{array}$ & $1,5,10,15$ \\
$\begin{array}{l}\text { Coagulation temperature } \\
\left({ }^{\circ} \mathrm{C}\right)\end{array}$ & 25 \\
\hline
\end{tabular}

\subsection{Membrane Characterizations}

Field emission scanning electron microscope (FESEM) (JEOL JSM6700F) was used to examine the morphology of the spun PVDF hollow fiber composite membrane by standard methods. The membrane samples were immersed in liquid nitrogen and fractured carefully. The samples were then dried in vacuum oven and coated with sputtering platinum before testing. The FESEM micrographs of crosssection and inner skin layer were taken at various magnifications. It produced photographs at the analytical working distance of $10 \mathrm{~nm}$.

The static contact angle of membrane was measured by the sessile drop method using a DropMeter A-100 contact angle system (Maist Vision Inspection \& Measurement Co. Ltd.) to characterize the membrane wetting 
behaviour. A water droplet of $3 \mu \mathrm{L}$ was deposited on the dry membrane using a microsyringe. A microscope with a long working distance $6.5 \mathrm{x}$ objectives was used to capture image.

Asymmetric porous membranes were characterized by determination of porosity and average pore radius. The membrane porosity, $\varepsilon$, was defined as the volume of the pores divided by the total volume of the porous membrane. The membrane porosity was calculated using the following equation,

$$
\varepsilon=\frac{\frac{\left(w_{1}-w_{2}\right)}{\rho_{W}}}{\frac{\left(w_{1}-w_{2}\right)}{\rho_{W}}+\frac{w_{2}}{\rho_{P}}} \times 100
$$

where $\varepsilon$ is the porosity of the membrane (\%), $w_{1}$ the weight of wet membrane $(\mathrm{g}), w_{2}$ the weight of dry membrane $(\mathrm{g}), \rho_{\mathrm{p}}$ the density of the polymer $\left(\mathrm{g} / \mathrm{cm}^{3}\right)$ and $\rho_{\mathrm{w}}$ is the density of water $\left(\mathrm{g} / \mathrm{cm}^{3}\right)$.

To prepare the wet and dry membranes, five spun hollow fibers with the length of $25 \mathrm{~cm}$ were selected after solvent was exchanged in tap water for 3 days. The fibers were immersed into the isopropanol for 3 days and distilled water for 3 days. The remained water in the inner surface was removed using air flow, before weighing the membranes. The wet membranes were dried in vacuum oven for $12 \mathrm{~h}$ at $40^{\circ} \mathrm{C}$ and weighted.

Average pore radius, $r_{m}$, was determined by filtration velocity method, in which pure water flux of the wet membrane was measured by applying pressure $(0.1 \mathrm{MP})$ for a limited period $(20 \mathrm{~h})$. It represents the average pore size along the membrane thickness $(\ell)$ that was the difference between external radius and internal radius of the hollow fiber membrane. The test module containing 60 fibers with the length of $35 \mathrm{~cm}$ was used to determine water permeability. According to Guerout-Elford-Ferry equation, $r_{\mathrm{m}}$ could be calculated:

$$
r_{\mathrm{m}}=\sqrt{\frac{(2.9-1.75 \varepsilon) x 8 \eta \ell Q}{\varepsilon x A x \Delta P}}
$$

where $\eta$ is water viscosity $\left(8.9 \times 10^{-4}\right.$ $\mathrm{Pa} s), \ell$ is the membrane thickness $(\mathrm{m})$, $\Delta P$ is the operation pressure $(0.1 \mathrm{MPa})$, $\varepsilon$ is the porosity of the membrane (\%), $Q$ is volume of permeate water per unit time $\left(\mathrm{m}^{3} \mathrm{~s}^{-1}\right), A$ is effective area of membrane $\left(\mathrm{m}^{2}\right)$.

\subsection{Permeation Flux and Rejection of Palm Oil Wastewater Measurements}

The permeation flux and rejection of PVDF hollow fiber membranes were measured by submerged ultrafiltration experimental equipment at various $\mathrm{pHs}$ of the feed solution (4.0 to 9.7). The equipment is shown schematically in Figure 1.



Figure 1 Schematic diagram of membrane process: $\left(\mathrm{v}_{1}, \mathrm{v}_{2}\right)$ feed flow, $\left(\mathrm{P}_{1}\right)$ pump, $\left(\mathrm{P}_{2}\right)$ compressor, (S) sparger, (M) membrane module, $\left(\mathrm{T}_{2}\right)$ membrane reservoir, (QC) flow control, (PC) pressure control, (LC) level control, (LI) level indicator 
An in-house assembled membrane module, with a filtration area of 12.48 $\mathrm{cm}^{2}$, was submerged in prepared suspension in a membrane reservoir with a volume of $15 \mathrm{~L}$. A cross-flow stream was produced by air bubbling generated by a diffuser situated underneath the submerged membrane module for mechanical cleaning of the membrane module. The air bubbling flow rate per unit projection membrane area was set constantly to $1.8 \mathrm{~L} / \mathrm{min}$ in order to maintain proper turbulence. The filtration pressure was supplied by a vacuum pump and controlled by a needle valve at 0.5 bar. Permeate flow rate were continually recorded using flow meter.

The rejection test was carried out with synthetic palm oil wastewater with a mixed liquor suspended solids (MLSS) concentration of $3 \mathrm{~g} / \mathrm{L}$ and various $\mathrm{pH}$ values (from 4.0 to 9.7 ). All experiments were conducted at $25^{\circ} \mathrm{C}$ using a vacuum pump. Firstly, the pure water permeation flux $\left(J_{w}\right)$ was measured at reduced pressure $(0.5$ bar) on the permeate side. Then, the permeation flux for the palm oil wastewater $\left(J_{R}\right)$ and rejection $(R)$ were measured also at the reduced pressure on the permeate side.

Membrane performance was tested with a self-made U-shape membrane module having about $12.48 \mathrm{~cm}^{2}$ of membrane surface area. Pure water permeation rate was measured after the steady state was reached, and the flux was calculated as

$$
\mathrm{F}=\frac{V}{A t}
$$

where $F$ is the pure water flux $\left(1 / \mathrm{m}^{2} \mathrm{~h}\right)$, $V$ is the permeate volume (1), $A$ is the membrane surface area $\left(\mathrm{m}^{2}\right)$, and $t$ is the time (h).

Rejection $(R)$ was measured using the synthetic palm oil wastewater after the pure water test. The synthetic palm oil wastewater was in-house produced and consisted of fresh water, hydraulic oil, diesel fuel, surfactant, and carbon black in a proper composition, having mixed liquor suspended solid (MLSS) of $3 \mathrm{~g} / \mathrm{l}$ and UV absorption at a wavelength of $2.6 \mathrm{~cm}^{-1}$. The rejection was calculated as

$$
\mathrm{R}=\left(1-\frac{c_{p}}{c_{f}}\right) \times 100
$$

where $R$ is the rejection ultrafiltration process $(\%), c_{\mathrm{p}}$ is the concentration of the permeate $(\%)$ and $c_{\mathrm{f}}$ is the concentration of the feed $(\%)$.

\subsection{RESULTS AND DISCUSSION}

\subsection{Morphological Studies of Composite Membranes}

Fabricated using dry-wet jet spinning method with three different batches of fibers for each dope composition. The morphologies of the membrane were studied by FESEM. Figure 2 shows the FESEM micrographs of the PVDF hollow fiber composite membranes prepared using different concentrations of $\mathrm{TiO}_{2}$. Improvement of membrane morphology is observed for addition of a small amount of $\mathrm{TiO}_{2}$ nanoparticles. $\mathrm{TiO}_{2}$ nanoparticles have high specific areas and hydrophilicity, which will affect the mass transfer during the spinning process.

The cross-sectional images for all hollow fibers consist of finger-like macrovoids extending from both inner and outer wall of the hollow fiber, and an intermediate sponge-like layer. The thickness of the sponge-like layer decreases initially with an increase in $\mathrm{TiO}_{2}$ concentration. However, with a further increase in $\mathrm{TiO}_{2}$ concentration the thickness of sponge-like layer starts to increase (Figures $2 \mathrm{a}$ to $2 \mathrm{c}$ ). This phenomenon can be explained by the kinetic effect on the rate of solvent- 
nonsolvent exchange in the phase inversion process. At lower $\mathrm{TiO}_{2}$ concentration, an increase in the amount of hydrophilic $\mathrm{TiO}_{2}$ tends to draw more water into the polymer dope, resulting in an increase in the length of finger-like macrovoids and decrease in the thickness of the intermediate sponge-like layer. Whereas at higher concentrations of $\mathrm{TiO}_{2}$, an increase in $\mathrm{TiO}_{2}$ concentration increases the viscosity of the polymer dope, decreasing the rate of water intrusion into the polymer dope, which results in the shorter finger-like macrovoids and thicker intermediate sponge-like layer.

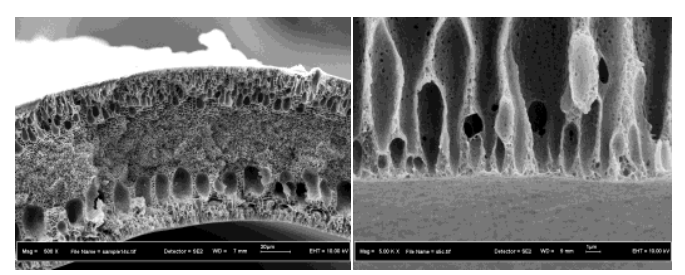

(a)

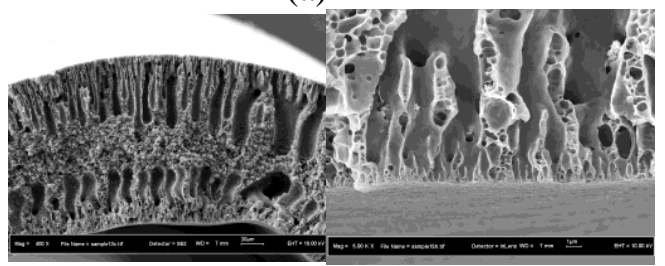

(b)

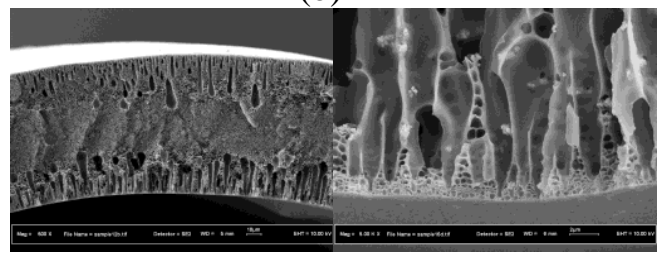

(c)

Figure 2 The cross-sectional and outer surface images of hollow fibers (Mag. 800x), (a) PTL-0, (b) PTL-10 and (c) PTL-20

\subsection{Porosity and Hydrophilicity Studies of PVDF Membranes}

Different concentrations of $\mathrm{TiO}_{2}$ nanoparticles were employed to fabricate membranes. The membranes were characterized in terms of surface hydrophilicity (contact angle), average pore radius $(r)$ and effective porosity $(\varepsilon)$. The results are shown in Table 3.

Surface hydrophilicity is one of the most important properties of membranes which could affect the flux and antifouling ability of membrane [21-24]. As presented in Table 3, contact angle decreased significantly with increasing $\mathrm{TiO}_{2}$ content up to 1.95 wt.\% (PTL-10). The decreased contact angle indicates the increase in hydrophilicity, which seems natural considering high hydrophilicity of $\mathrm{TiO}_{2}$ particles due to the presence of hydroxyl group. However, a further increase of $\mathrm{TiO}_{2}$ concentration results in an increase in contact angle (decrease in hydrophilicity). This is most likely due to the agglomeration of $\mathrm{TiO}_{2}$ nanoparticles, which reduces the area of contact of hydroxyl groups carried by the $\mathrm{TiO}_{2}$ nanoparticles.

Table 3 lists the porosity and average pore size of prepared membranes, which showed a good porosity in the range of 63 to $85 \%$, which can be attributed to the low polymer concentration in the spinning dope and additives used. The porosity increased with an increase in $\mathrm{TiO}_{2}$ content up to (PTL-10) but then decreased with further increase in $\mathrm{TiO}_{2}$ content. This co insides with the change of the intermediate sponge layer thickness (initial decrease and then increase with further increase in $\mathrm{TiO}_{2}$ content) as shown by the FESEM images.

The properties of prepared composite membranes at different air gap length was studied in term of pure water flux. As can be seen from Figure 3 , it is noticed that the increase of air gap length decreased the pure water permeation and also decreased in wall thickness. Generally, the skin layer thickness affects the membrane performance as well. On the other hand, the average pore radius decreased with increasing $\mathrm{TiO}_{2}$ concentration in the 
spinning dope due to interaction between $\mathrm{TiO}_{2}$ and PVDF.

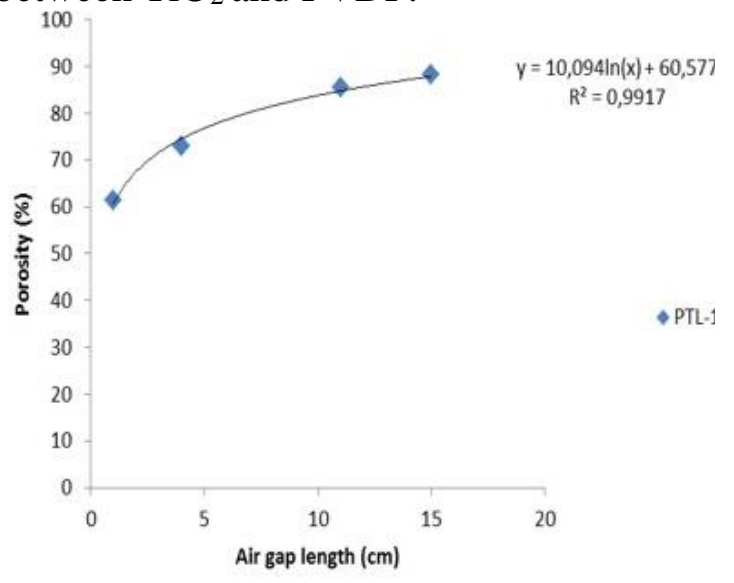

Figure 3 Porosity of composite membranes versus air gap

A small proportion of $\mathrm{TiO}_{2}$ nanoparticles in the dope obtained the existed interfacial stresses between the polymer and $\mathrm{TiO}_{2}$ nanoparticles, which formed of the organic phase shrinkage during the demixing process. However, the added higher $\mathrm{TiO}_{2}$ concentration are blocked the pores and formed a denser cross-sectional substructure, consequently decreasing the average pore size. The changes in the average pore size corresponds with the change in the porosity. This seems also natural considering the larger size of the finger-like pores as compared to the sponge-like pores.

\subsection{Effect of Surface Properties on Permeability and Rejection}

As shown in Table 3, PTL-10 showed a maximum flux of $82.49 \mathrm{~L} / \mathrm{m}^{2} \mathrm{~h}$ for $\mathrm{TiO}_{2}$ concentration of $1.95 \mathrm{wt} \%$ (PTL10). The rejection values demonstrated the similar trend to the flux, showing the maximum value of $98.8 \%$ at 1.95 wt.\% $\mathrm{TiO}_{2}$ concentration. It is interesting to note that the observed trend is contrary to the trade-off effect, by which rejection should decrease as flux increases. It is easy to understand that the flux shows a maximum value for the membrane PTL-10, since both porosity and pore size become the highest for this particular membrane. The maximum in rejection occurring at the same $\mathrm{TiO}_{2}$ concentration, on the other hand, can be explained by the trend observed in the surface hydrophilicity. Contact angle is the lowest, meaning hydrophilicity is the highest for PTL-10. Most likely, water is preferentially transported through the membrane as compared to the hydrophobic components of the palm oil waste water when the membrane surface is hydrophilic, thus the highest rejection of oily components corresponds to the highest surface hydrophilicity of the membrane. $\mathrm{TiO}_{2}$ particles on the membrane surface reduced the interaction between contaminants and the membrane surface. The increased membrane hydrophilicity and membrane pore size with lower $\mathrm{TiO}_{2}$ concentration $(\leq 1.95$ wt.\%) could attract water molecules inside composite membrane; facilitated their penetration through membrane, enhancing flux and rejection. The antifouling properties of PVDF ultrafiltration membranes can be evaluated by the ratio of palm oil wastewater flux $\left(J_{R}\right)$ and pure water flux $\left(J_{W}\right)$. For the higher antifouling submerged UF membrane, the feed of palm oil wastewater would cause a smaller flux loss and the ratio $\left(J_{R} / J_{W}\right)$ would become higher.

Table 3 Properties of fabricated membrane

\begin{tabular}{lccccc}
\hline Membrane & $\begin{array}{c}\text { Contact angle, } \\
\text { (s.d.) }\end{array}$ & $\begin{array}{c}\text { Average pore } \\
\text { size(nm), } \\
(\mathbf{s . d .})\end{array}$ & $\begin{array}{c}\text { Overall porosity } \\
(\boldsymbol{\%})\end{array}$ & $\begin{array}{c}\text { Flux, } \\
\left(\mathbf{L} / \mathbf{m}^{2} . \mathbf{h}\right)\end{array}$ & Rejection (\%) \\
\hline PTL-0 & $81.05(0.79)$ & $28.0(1.44)$ & 66.96 & 74.04 & 62.56 \\
PTL-10 & $47.33(1.44)$ & $34.05(1.01)$ & 85.41 & 84.20 & 98.83 \\
PTL-20 & $57.67(0.87)$ & $26.07(0.97)$ & 63.26 & 79.83 & 91.71 \\
\hline
\end{tabular}


Figure 4 also shows that the ratio $\left(J_{R} / J_{W}\right)$ first increased and reached the maximum at $\mathrm{TiO}_{2}$ concentration of $1.95 \mathrm{wt} \%$. This is also due to the highest hydrophilicity at this particular $\mathrm{TiO}_{2}$ concentration, science high hydrophilicity reduces the interaction between the hydrophobic contaminants and the membrane surface, effectively improving the antifouling properties. This clearly demonstrates the antifouling properties of $\mathrm{TiO}_{2}$ when it is added to PVDF composite membranes.

\subsection{Effect of $\mathrm{pH}$ on Flux Performances}

The flux is also strongly influenced by the $\mathrm{pH}$ value of feed solution. The permeate flux of a PVDF composite membrane (PTL-10) at various $\mathrm{pH}$ values of $4.0,5.0,6.9,8.1$ and 9.7 during palm oil wastewater treatment is presented in Figure 4. The steady flux increased sharply with increasing $\mathrm{pH}$ from 4.0 to 6.9 and the increase became less steep from $\mathrm{pH} 6.9$ to 9.7 due to the chemical interactions between the of $\mathrm{pH}$ on the zeta potential. Due to the presence of the surfactant, the oil droplet is negatively charged. Therefore, the electrostatic affinity accelerates fouling formation since droplets adsorb onto the membrane surface and penetrate into the membrane pores, lowering the steady permeate flux at $\mathrm{pH}$ 4.0. Thus, at lower $\mathrm{pH}$ values, the increase in suspended solid aggregation, and in turn flux reduction caused by formation of a thicker suspended solid deposit, is likely due to reduction of electrostatic repulsion. However, from $\mathrm{pH} 6.9$ to 9.7 the charged of membrane surface is negative. Repulsive forces reduce the adsorption of foulant and limit the membrane pore blocking that increases permeability and results in higher permeate flux.
As discussed by Yuan and Zydney [25], the initial flux decline during membrane filtration is due to the physical deposition of large suspended solid aggregates on the membrane surface. As known, it was reported that lower $\mathrm{pH}$ induced a lower flux. Moreover, the slightly flux decline seen from pH 6.9 to 9.7 was caused by foulant deposition on the upper surface of the membrane at higher $\mathrm{pH}$. Briefly, the flux is higher at higher $\mathrm{pH}$ values.

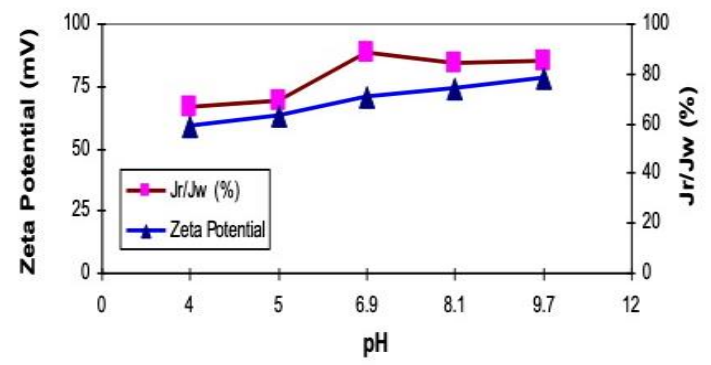

Figure 4 Effect of solution $\mathrm{pH}$ and zeta potential on the steady flux during filtration by membrane PTL-10

\subsection{CONCLUSION}

The amounts of $\mathrm{TiO}_{2}$ were varied as non-solvent additives in the spinning dopes to improve the phase-inversion rate and provide porous asymmetric membranes with improved structure for palmoil produced wastewater treatment. Various characterizations and measurements techniques such as membrane structure, surface wettability, porosity, average pore size, and permeability were utilized to evaluate fine structural details of the membrane and membrane performance. Palm oil produced wastewater filtration was conducted through prepared PVDF composite membranes. FESEM analysis indicated that PVDF concentration of 19 wt. \% had suppressed both inner and outer membrane surface finger-like macrovoids with slightly denser skin layer which decreased mass transport resistance. Addition of 1.95 wt.\% of $\mathrm{TiO}_{2}$ nanoparticles resulted in smaller 
nanoparticles which in turn achieved higher hydrophilicity, small pore size, and high porosity. Permeability test illustrated that $\mathrm{TiO}_{2}$ nanoparticles affected the PVDF composite membranes performance remarkably. Significantly higher flux and rejection of palm oil wastewater were observed. Furthermore, the steady permeate flux achieved the decreased results at $\mathrm{pH}$ 6.7 to 9.7 slightly. These values also reflected the obtained thicker suspended solid deposit on the membrane outer surface charged membrane surface and oil droplets.

\section{ACKNOWLEDGEMENT}

The authors would like to thank Ministry of Higher Education of Republic Indonesia for providing facilities and guides to complete this project.

\section{REFERENCES}

[1] N. F. Jasney, A. L. Ahmad, S. Ismail. 2014. Ultrafiltration Membrane for POME Treatment: Comparison Physical \& Chemical Cleaning Process. J. Teknologi. 69(4): 77-83.

[2] N. H. Zainal, N. F.Jalani, R. Mamat, A. A. Astimar. 2017. A Review on the Development of Palm Oil Mill Effluent (POME) Final Discharge Polishing Treatments. J. Oil Palm Research. 29(4): 528-540.

[3] M. Said, A. W. Mohammad, M. T. Mohd Nor, H. A. Hasan. 2014. Palm Oil Mill Effluent Treatment through Combined Process Adsorption and Membrane Filtration. Memb. Water Treat. 5(3): 207-219.

[4] A. W. Zularisam, A. F. Ismail, M. R. Salim. 2006. Behavior of
Natural Organic Matter (NOM) in Membrane Filtration for Surface Water Treatment: A Review. Desalination. 194(1-3): 211-231.

[5] Q. Li, Z. L. Xu, L. Y. Yu. 2010. Effect of Mixed Solvents and PVDF Types on Perfomances of PVDF Microporous Membranes. J. Appl. Polym. Sci. 115: 22772287.

[6] M. Khayet, T. Matsuura. 2001. Preparation and Characterization of Polyvinylidene Fluoride Membranes for Membrane Distillation. Ind. Eng. Chem. Res. 40: 5710-5718.

[7] M. Khayet, C. Y. Feng, K. C. Khulbe, T. Matsuura. 2002. Study on the Effect of a Nonsolvent Additive on the Morphology and Performance of Ultrafiltration Hollow Fibre Membranes. Desalination. 148: 321-327.

[8] S. Chabot, C. Roy, G. Chowdhury, T. Matsuura. 1997. Development of Poly(vinylidene fluoride) Hollow Fiber Membranes for the Treatment of Water/Organic Vapor Mixtures. J. Apply. Polym. Sci. 65: 12631270.

[9] X. C. Cao, J. Ma, X. H. Shi, Z. J. Ren. 2006. Effect of $\mathrm{TiO}_{2}$ Nanoparticle Size on the Performance of PVDF Membrane. Apply. Surf. Sci. 253: 2003-2010.

[10] T. H. Bae, I. C. Kim, T. M. Tak. 2006. Preparation and Characterization of Foulingresistant $\mathrm{TiO}_{2}$ Self-assembled Nanocomposite Membrane. $J$. Membr. Sci. 275: 1-5.

[11] A. Bottino, G. Capanelli, S. Munari, A. Turturro. 1998. High Performance Ultrafiltration Membranes Cast from $\mathrm{LiCl}$ 
Doped Solution. Desalination. 68: 167-177.

[12] W. J. Lau, A. F. Ismail. 2009. Theoritical Studies on the Morphology and Electrical Properties of Blended PES/SPEEK Nanofiltration Membranes using Different Sulfonation Degree of SPEEK. $J$. Membr. Sci. 334: 30-42.

[13] K. Khayet, C. Y. Feng, K. C. Khulbe, T. Matsuura. 2002. Preparation and Characterization of Polyvinylidene Fluoride Hollow Fiber Membranes for Ultrafiltration. Polymer. 43: 3879-3890.

[14] M. L.Yeow, Y. Liu, K. Li. 2005. Preparation of Porous PVDF Hollow Fibre Membrane via a Phase Inversion Method Using Lithium Perchlorate $\left(\mathrm{LiClO}_{4}\right)$ as an Additive. J. Membr. Sci. 258: 16-22.

[15] X. Cao, J. Ma, X. Shi, Z. Ren. 2006. Effect of $\mathrm{TiO}_{2}$ Nanoparticle Size on the Performance of PVDF Membrane. Appl. Surf. Sci. 253: 2003-2010.

[16] Y. J. Wang, D. J. Kim. 2007. Crystallinity, Morphology, Mechanical Properties and Conductivity Study of In Situ Formed $\quad \mathrm{PVDF} / \mathrm{LiClO}_{4} / \mathrm{TiO}_{2}$ Nanocomposite Polymer Electrolytes. Electrochimica Acta. 52: 3181-3189.

[17] E. Fontananova, J. C. Jansen, A. Cristiano, E. Curcio, E. Drioli. 2006. Effect of Additives in the Casting Solution on the Formation of PVDF Membranes. Desalination. 192: 190-197.

[18] Y. S. Li, L. Yan, C. B. Xiang, L. J. Hong. 2006. Treatment of Oily Wastewater by Organicinorganic Composite Tubular Ultrafiltration (UF) Membranes. Desalination. 196: 76-83.
[19] S. J. Oh, N. Kim, Y. T. Lee. 2009. Preparation and Characterization of $\mathrm{PVDF} / \mathrm{TiO}_{2}$ Organic-inorganic Composite Membranes for Fouling Resistance Improvement. J. Membr. Sci. 345: 13-20.

[20] D. Wang, K. Li, W. K. Teo. 2000. Porous PVDF Asymmetric Hollow Fiber Membranes Prepared with the Use Small Molecular Additives. J. Membr. Sci. 178: 13-23.

[21] A. W. Zularisam, A. F. Ismail, M. R. Salim, M. Sakinah. 2007. Roles of Natural Organic Matter (NOM) Fractions on Fouling Characteristics and Flux Recovery of Ultrafiltration Membrane. Desalination. 212: 191-208.

[22] S. Judd. 2006. The MBR Book, Principles and Applications of Membrane Bioreactors in Water and Wastewaters Treatment. Elsevier, Oxford.

[23] A. F. Ismail, W. J. Lau. 2009. Influence of Feed Conditions on the Rejection of Salt and Dye in Aqueos Solution by Different Charactheristics of Hollow Fiber Nanofiltration Membranes. Desalination. 6: 281-288.

[24] M. M. Rahman. 2004. Treatment of Palmoil Wastewater Using Crossflow Membrane Bioreactor (CF-MBR). Ph.D Thesis. King Fahd University of Petroleum and Minerals.

[25] C. Y. Chiang, M. J. Reddy, P. P. Chu. 2004. Nano-tube Composite PVDF/LiPF6 Solid Membranes. Solid States Ionics. 175: 631-635.

[26] W. Yuan, A. L. Zydney. 2000. Humic Acid Fouling during Ultrafiltration. J. Environ. Sci. Technol. 34(23): 5043-5050. 